\title{
QoE-aware Power Management in Vehicle-to-Grid Networks: a Matching-theoretic Approach
}

\author{
Ming Zeng, Supeng Leng, Member, IEEE, Yan Zhang, Senior Member, IEEE, and Jianhua He, Senior \\ Member, IEEE
}

\begin{abstract}
Frequency, time and places of charging and discharging have critical impact on the Quality of Experience (QoE) of using Electric Vehicles (EVs). EV charging and discharging scheduling schemes should consider both the QoE of using EV and the load capacity of the power grid. In this paper, we design a traveling plan-aware scheduling scheme for EV charging in driving pattern and a cooperative EV charging and discharging scheme in parking pattern to improve the QoE of using $\mathrm{EV}$ and enhance the reliability of the power grid. For traveling planaware scheduling, the assignment of EVs to Charging Stations (CSs) is modeled as a many-to-one matching game and the Stable Matching Algorithm (SMA) is proposed. For cooperative EV charging and discharging in parking pattern, the electricity exchange between charging EVs and discharging EVs in the same parking lot is formulated as a many-to-many matching model with ties, and we develop the Pareto Optimal Matching Algorithm (POMA). Simulation results indicates that the SMA can significantly improve the average system utility for EV charging in driving pattern, and the POMA can increase the amount of electricity offloaded from the grid which is helpful to enhance the reliability of the power grid.
\end{abstract}

Index Terms - vehicle-to-grid, power charging and discharging, matching theory, QoE, preference.

\section{INTRODUCTION}

D RIVING range and charging of Electric Vehicle (EV) are key concerns when people choose either EV or gasoline car. The short driving range of EVs, long charging time and shortage of Charging Stations (CSs) are among the major reasons that put people off the EVs. Quality of Experience (QoE) as a new Quality of Service (QoS) metric in Vehicle-toGrid (V2G) networks, express the degree of satisfaction with the State Of Charge (SOC) and charging cost of using an EV. How to effectively improve the QoE of using EV is one of the big challenges faced by the EV industry. On the other hand, with the increasing penetration of EVs into vehicle market (5 million EV in China by 2020 predicted by McKinsey [1]), tremendous electricity demand due to EV charging is expected

Manuscript received February 29, 2015. Accepted for publication September 19, 2015. Copyright (c) 2016 IEEE. Personal use of this material is permitted. However, permission to use this material for any other purposes must be obtained from the IEEE by sending a request to pubs-permissions@ieee.org.

This work is supported by the National Natural Science Foundation of China (61374189), the Fundamental Research Funds for the Central Universities (ZYGX2013J009), partially supported by the projects 240079/F20 funded by the Research Council of Norway, the project IoTSec-Security in IoT for Smart Grids, with number $248113 / 070$ part of the IKTPLUSS program funded by the Norwegian Research Council.

M. Zeng and S. Leng (corresponding author) are with the School of Communication and Information Engineering, University of Electronic Science and Technology of China, Chengdu, Sichuan Province, 611731 China (Phone: +86 028 618-305-20; Fax: +86028 618-305-20; e-mail: spleng@uestc.edu.cn).

Y. Zhang is with University of Oslo, Norway. He is also with Simula Research Laboratory, Norway. (e-mail: yanzhang@ieee.org ).

J. He is with Aston University, Birmingham, B4 7ET, UK (e-mail: j.he7@aston.ac.uk). to challenge the current power grid. As investigated in [25], EV users are used to charge their EVs immediately after they arrive in the workplaces at around 9:00am or at home at around 6:00pm. Similar charging patterns of large-scale of EVs in the peak period of power grid load will significantly increase the burden of the power grid and reduce the reliability of the power grids. In addition, charging in peak hours is not cost effective as the electricity in the peak period is more expensive.

Motivated by the above EV charging problems, we propose QoE aware power management solutions. We design a traveling plan-aware scheduling scheme for EV charging in driving pattern, and a cooperative EV charging and discharging scheme in parking pattern. Both schemes can improve the QoE of using EVs, the utilization of CSs and the reliability of the power grid. In the traveling plan-aware scheduling scheme, we consider not only the benefits of CSs and power grid as part of the optimization objective, but also treat the QoE of using EVs with high importance. We incorporate EV traveling plans into the charging scheduling scheme, so that EVs can be properly assigned to CSs located along their travel routes, in order to save additional driving time and energy. In the cooperative charging and discharging scheme, we propose an electricity exchange system for EVs parking in the same parking lot and devise an effective electricity trading scheme. In this way, the impact on the stability of the grid due to EV charging can be significantly reduced. The unnecessary cost of power delivery between parking lots and the grid can be avoided. Moreover, the costs of EV charging are decreased and discharging EVs are incentivized with profits made by selling surplus electricity to other EVs. Both the EV charging scheduling problem in driving pattern and the cooperative charging and discharging problem in parking pattern are formulated under the matching theoretic framework. Low complexity algorithms are developed to solve the problems.

It is noted that in the current work the charging problems for EVs in two patterns are studied under the framework of matching theory but as independent optimization problems. We believe these two problems can be connected under a joint optimization framework. One simple extension is let charging EVs select electricity supplier between CSs and discharging EVs. With such extension, charging EVs are more likely to charge at lower charging cost while the utilization of CSs can be more flexible and efficient. Moreover, the stability of the power grid can be further guaranteed. The complexity of such joint optimization problem will increase and solutions with tractable complexity need to be explored. This joint optimization problem is interesting but is left as our future work. This work focuses on getting insights into the EV charging problem with preference considered and find design guidelines for the separate charging optimization problems. 
Our main contributions can be summarized as follows.

- Traveling information of EVs are taken into account in the design of EV charging scheduling scheme in driving pattern. The optimization objective is to maximize the total system utility of both EVs and CSs. The problem is formulated as a many-to-one matching model and a stable matching algorithm is developed to solve the problem with tractable complexity.

- Cooperative EV charging and discharging in parking pattern is proposed to help offload electricity demand from the power grid and offer incentives to EVs in the parking lot. The matching between charging EVs and discharging EVs is formulated as a many-to-many matching model with ties due to their random parking time, different valuation of electricity unit price, variable amount of electricity demand and supply.

- An extended dynamic version of network maximum flow algorithm is designed to find a Pareto optimal matching between charging EVs and discharging EVs. The optimization objective in parking pattern is to maximize the trading volume of electricity to enhance the reliability of the power grid.

The rest of this paper is organized as follows. Section II presents the related research work. In Section III, we propose the traveling plan-aware scheduling model for EV charging in driving pattern and formulate it as a college admission problem in the matching theory. The Stable Matching Algorithm (SMA) is proposed to find a stable matching between charging EVs and CSs. In Section IV, the cooperative EV charging and discharging problem in parking pattern is formulated as a many-to-many matching problem with ties. The Pareto Optimal Matching Algorithm (POMA) is proposed to seek a Pareto optimal matching between discharging EVs and charging EVs. Performance evaluation is presented in Section V. Finally, Section VI concludes the paper.

\section{RELATED WORK}

Coordination of EV charging has been widely investigated because of the increasing penetration of EVs. Reference [3] examines the impact of different charging strategies and charging power ratings on the distribution grid of a residential building. Reference [4] studies EV charging scheduling problem when stochastic wind power is utilized to be the energy source. The authors in reference [5] derive a generic formulation of optimal EV charge scheduling with nonlinear state-of-charge curve and uncertainty of the price of electricity. Reference [6] leverages the model predictive control based method to design a dynamic charging and mode switching strategy to optimize the driving cost of the EV driver. Reference [7] proposes a stochastic dynamic programming solution method to reduce average charging costs of EVs. Reference [8]-[11] reported on game theoretical approaches to the EV charging problems. The work [8] presents a multi-stage approach to the placement of charging stations, where a nested logit model is used to forecast the charging demand and a Bayesian game among service providers is formulated to derive the optimal station placement strategy. Reference [9] applies a non-cooperative game model with coupled constraints to charging scheduling problem of parking-lot EVs. Reference [10] proposes an integrated EV charging navigation framework modeled by a hierarchical game model. Competition between fast charging stations is formulated as a non-cooperative game model while the strategies for EVs in choosing charging station is solved by a multiple evolutionary game approach. Reference [11] formulates the competition between charging of EVs as a game of chicken, and investigates the inefficiency of Nash equilibria.

Most literatures about EV traveling and charging scheduling optimization aim to minimize waiting time at charging station [22] or total travel time [23], or maximize the utilization rate of charging infrastructure [24]. Little literature takes into account the preference of charging EVs and seeks to maximize the total utility of both charging EVs and CSs. However, the QoE of using EVs, which is reflected by the preferences of charging $\mathrm{EVs}$, has great influence on people's purchase decision on EVs or gasoline vehicles. Matching theory is particularly suitable for scheduling problems to improve the QoE of charging. First, in matching theoretic approaches, both electricity sellers and charging EVs are able to configure their preferences based on utilities during the process of charging resource allocation. Second, matching theoretic approaches can be implemented either in centralized way or distributed way with tractable complexity. The problems solved by matching theory are usually divided into three types: one-to-one matching, one-tomany matching and many-to-many matching. Alvin E. Roth and Lloyd Shapley proposed the deferred acceptance algorithm [17] for one-to-one stable marriage matching and were awarded Nobel economic prize in Year 2012. The convergence of deferred acceptance algorithms to stable matching is guaranteed irrespective of the order of proposals and without any synchronization. Therefore, deferred acceptance algorithms can be implemented in a distributed manner without central information collectors. Meanwhile, the convergence of deferred acceptance algorithms to stable matching is polynomial [12].

We apply the one-to-many matching model for EV charging scheduling problem in driving pattern and many-to-many matching model for the cooperative charging and discharging system we propose. Stable Matching Algorithm (SMA) based on classic deferred acceptance algorithm is proposed for EV charging scheduling problem in driving pattern. However, specific method to solve many-to-many matching problems has not been found yet. Thus, we design the Pareto Optimal Matching Algorithm (POMA) to find a Pareto optimal matching between charging EVs and discharging EVs in parking pattern.

\section{Traveling Plan-Aware Scheduling For EV ChARGING IN DRIVING PATTERN}

In this section, we consider charging scheduling of EVs at CSs. It is foreseeable that CSs in the future will be distributed ubiquitously in cities, but the capacity of each CS is constrained. Each CS can only offer limited charging interfaces to a few charging EVs to charge simultaneously. Since the overall capacity of the CSs is highly limited comparing with the electricity demand of EVs, we believe that the EVs can be motivated to request charging service at the CSs in advance to avoid queuing at the CSs. It is expected that some EVs do not make charging reservation when arriving at the CSs. There can be options to deliver charging services to EVs without charging reservation depending on their priorities. For 
simplicity, we consider the scheduling problem of the charging interfaces at CSs purely for the EVs which make charging requests in advance. It is noted that the work presented in this paper is easy to be extended to the scheduling problem when the EVs without charging reservation are considered by the CSs.

Our main motivation of the charging scheduling design at CSs is to take into account the traveling plans and preferences of the EV drivers and improve the QoE of EV charging. One example is shown in Figure 1. We assume that $E V_{1}$ has a travel plan of passing by $C S_{1}$ at around 9:00am and $7 \mathrm{kWh}$ electricity is demanded at $C S_{1}$, and then passing by $C S_{2}$ and $C S_{4}$ at around 9:30am and 11:00am, respectively. Thus $C S_{1}, C S_{2}$ and $C S_{4}$ are the CS candidates for $E V_{1}$. Similarly according to the travelling information of $E V_{2}$, the CSs $C S_{3}$ and $C S_{4}$ are on the route of $E V_{2}$ and are candidates of $E V_{2}$. We propose the Stable Matching Algorithm (SMA) to determine proper charging stations according to traveling plans and preference lists of EVs. Based on the SMA, a stable assignment to CSs for EV charging is given, then EVs follow the scheduling results to charge.

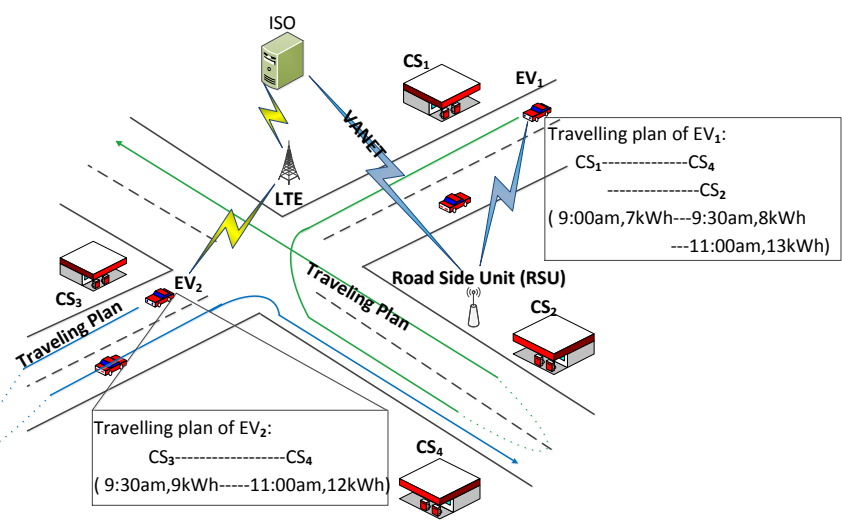

Figure 1: Traveling plan-aware scheduling for EV charging in driving pattern

\section{A. System Model}

In our proposed model, charging EVs and CSs interact under the assistance of the Independent System Operator (ISO) for signaling exchange as shown in Figure 2. Charging requests and scheduling decisions are transmitted through the cellular networks or Vehicle Ad hoc NETworks (VANET) [13] with seamless coverage [14]. The matching process between charging EVs and CSs is executed periodically. One matching period consists of three stages. Stage 1 is the charging request collection stage, while the matching process is executed in Stage 2 and in Stage 3 scheduling decisions are sent from CSs to EVs. The ISO specifies the length of Stage 1 and determines the time when matching results can be published. During Stage 2 or 3, if CSs receive new charging requests from other EVs rather than in Stage 1, those charging requests will be cached by CSs and will be processed until next matching period. The status of charging interfaces of CSs will be updated at the end of each matching period.

According to the above system operation description, it is noted that the scheduling decisions are made by CSs for each

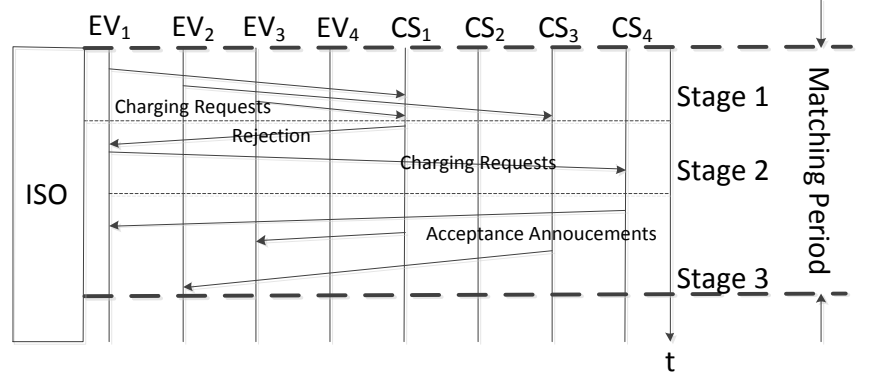

Figure 2: Timing diagram of matching period

individual matching period based on the charging requests received from the EVs. The scheduling process is independent of the matching periods. Therefore, we focus and design the scheduling process for one matching period. Without loss of generality, we suppose that in one general matching period there are $N$ CSs from the set of CSs $\mathcal{N}$, labeled by the index $i, i \in\{1,2, \ldots, N\} . C S_{i}$ can provide at most $K_{i}$ interfaces for EV charging simultaneously. The charging rate of each interface is assumed to be the same and fixed. We assume at the end of Stage $1 M$ charging EVs from the set of charging EVs $\mathcal{M}$ have sent charging requests to CSs. The index of each charging EV is $j, j \in\{1,2, \ldots, M\}$.

Let $\Omega$ be a $N \times M$ allocation matrix with element $a_{i j}=$ $\{0,1\} . a_{i j}=1$ means $E V_{j}$ is assigned to the $C S_{i}$, and $a_{i j}=$ 0 means no assignment is between $E V_{j}$ and $C S_{i}$. As one charging EV can only be assigned to one CS, while $C S_{i}$ could offer charging opportunities to at most $K_{i}$ EVs simultaneously. Thus, we have two constraints, i.e.,

$$
\begin{gathered}
\sum_{i=1}^{N} a_{i j} \leq 1 \\
\sum_{j=1}^{M} a_{i j} \leq K_{i} .
\end{gathered}
$$

To guarantee the QoE of EV charging, the utility of charging $\mathrm{EV}$ is considered from the following aspects. First, charging EVs prefer to charge at CSs along their traveling route and stations which can be reached with remaining electricity in their batteries. Second, drivers usually will specify the latest start charging time $t_{j}$ considering their itineraries. Third, the amount of electricity the CS could offer will also affect the utility of the charging EV. The EV will suffer potential risk if the amount of electricity irrigated from CS is inadequate. The EV may be out of power before reaching next available CS and need to be charged frequently. Hence, the utility function $U E_{i, j}$ that can be used to measure the QoE if $E V_{j}$ charges at $C S_{i}$ is defined as

$$
U E_{i, j}=Q_{j}^{i}-d_{j}^{i} * R_{j}-C\left(t_{j}^{i}\right)
$$

where $Q_{j}^{i}$ is the amount of electricity $C S_{i}$ sells to $E V_{j}$, $d_{j}^{i}$ is the distance $E V_{j}$ has to drive to $C S_{i}$ and back to its traveling route after charging completed, $R_{j}$ is the electricity consumption rate of $E V_{j}$, and $t_{j}^{i}$ is the arriving time of $E V_{j}$ at $C S_{i}$. The battery capacity of EV is limited, hence, the maximum amount of electricity EV $j$ need is denoted by $Q_{j}^{\max }$. The utility function of $E V_{j}$ should be higher if $C S_{i}$ 
could offer more electricity but not exceed $Q_{j}^{\max }$, meanwhile $C S_{i}$ should not be too far to reach. $C($.$) function is a step$ function representing the cost due to delay. It is probable that $E V_{j}$ will arrive at CS $j$ later than the expected time $t_{j}$ due to road congestion or other reasons. If the arriving time $t_{j}^{i}$ of $E V_{j}$ at CS $i$ is later than $t_{j}, C\left(t_{j}^{i}\right)=C$ where $C$ can be set by the driver of the EV to express his/her tolerance on the charging access delay, otherwise $C\left(t_{j}^{i}\right)=0$. Drivers of charging EVs are incentive rational, which implies,

$$
\begin{gathered}
a_{i j} U E_{i, j}>0, \forall i \in \mathcal{N}, j \in \mathcal{M} \\
Q_{j}^{i} \leq Q_{j}^{\max }, \forall i \in \mathcal{N}, j \in \mathcal{M}
\end{gathered}
$$

It is noted that three significant factors that affect the QoE of using EV are included in the utility definition of the charging EV. The QoE of using EV will be improved with less frequent charging, less electricity consumption for driving to the CSs and lower charging access delay.

For tractability, the unit price of electricity purchased at each CS is set to be identical. For higher utility, CSs prefer EVs which demand more electricity. Hence, the utility $U C_{i, j}$ of $C S_{i}$ offering charging opportunity to $E V_{j}$ has the following characteristic

$$
U C_{i, j}>U C_{i, j^{\prime}}, \text { for } Q_{j}^{i}>Q_{j^{\prime}}^{i}
$$

The objective of the system is to maximize the utilities of both charging EVs and CSs. $\beta$ is a weight parameter which adjusts the impact of charging EVs and CSs, i.e.,

$$
\max _{a_{i j}} \sum_{i \in \mathcal{N}} \sum_{j \in \mathcal{M}} a_{i j} U E_{i, j}+\beta \sum_{j \in \mathcal{M}} \sum_{i \in \mathcal{N}} a_{i j} U C_{i, j}
$$

Thus, the optimization problem is formulated as

$$
\begin{gathered}
\max _{a_{i j}} \sum_{i \in \mathcal{N}} \sum_{j \in \mathcal{M}} a_{i j} U E_{i, j}+\beta \sum_{j \in \mathcal{M}} \sum_{i \in \mathcal{N}} a_{i j} U C_{i, j} \\
\text { s.t. } \sum_{i=1}^{N} a_{i j} \leq 1, \sum_{j=1}^{M} a_{i j} \leq K_{i}, a_{i j}=\{0,1\} \\
Q_{j}^{i} \leq Q_{j}^{\text {max }}, \forall i \in \mathcal{N}, j \in \mathcal{M} \\
a_{i j} U E_{i, j}>0, \forall i \in \mathcal{N}, j \in \mathcal{M}
\end{gathered}
$$

The optimization problem expressed by Equation (8)-(11) is an Integer Linear Programming (ILP) problem. The computational complexity of ILP problems will increase exponentially with the scale of CSs and charging EVs [20], so we model the optimization problem as a college admission problem in matching theory [17].

\section{B. Many-to-One Matching Model}

The traveling plan-aware scheduling problem for EV charging in driving pattern can be modeled as a many-to-one matching game. First, we give the basic terminology in matching theory and then describe the proposed algorithm.

Definition 1: Matching

A matching $\Phi$ in the instance of EV charging scheduling is an assignment such that

- each EV is assigned to at most one CS in $\Omega$, i.e., $\Phi\left(E V_{j}\right) \in \mathcal{N} \bigcup\{\varnothing\}$, and $\left|\Phi\left(E V_{j}\right)\right|=\{0,1\}$,
- each CS is assigned to at most $K_{i}$ EVs in $\Omega$, i.e., $\Phi\left(C S_{i}\right) \in \mathcal{M} \bigcup\{\varnothing\}$, and $\left|\Phi\left(C S_{i}\right)\right|=\left\{0,1, \ldots, K_{i}\right\}$,

where $\Phi\left(E V_{j}\right)$ denotes the CS that is assigned to EV $j$, and $\Phi\left(C S_{i}\right)$ denotes the EV that is assigned to CS $i$.

Definition 2: Preference List

Each EV $j \in \mathcal{M}$ ranks a subset of CSs in $\mathcal{N}$ in strict descending order according to the utility function defined in equation (3) giving rise to its preference list $\mathbf{P L}_{j}$.

Definition 3: Stable Matching

A matching $\Phi$ is stable if and only if there are no two EVs $j$ and $j^{\prime}, \forall j, j^{\prime} \in \mathcal{M}$ that $\Phi(j)_{j} \prec \Phi\left(j^{\prime}\right)$ and $\Phi\left(j^{\prime}\right)_{j^{\prime}} \prec \Phi(j)$ [17], where $\Phi(j)_{j} \prec \Phi\left(j^{\prime}\right)$ means EV $j$ prefers CS assigned to EV $j^{\prime}$ to its assignment $\Phi(j)$, and so does EV $j^{\prime}$.

It is well known that Gale and Shapely proposed the acceptance deferring algorithm in [17] to solve the two-sided one-to-one matching problem with tractable complexity. We extend the classic algorithm to the many-to-one matching problem and propose the Stable Matching Algorithm (SMA). First, each EV calculates the utilities if it charges at different CSs. Then, those CSs with positive utilities are included in the the preference lists of corresponding EVs and are sorted in descending orders. Each EV first proposes to its favorite CS in the preference list. After receiving proposals from all EVs, each CS checks if the number of proposals in PROPOSE exceeds the number of its available interfaces. If CS $i$ does not receive so many charging requests, it will accept all, and cache them in IN_SUSPENCE ${ }_{i}$. Otherwise, CS $i$ will choose $K_{i}$ EVs which request most amounts of electricity and cache them, and reject the others. EVs which are rejected by CSs update the set of rejecting CSs REJ_STA. In the next iteration, EVs which have been rejected propose to the highest ranked CSs in their preference list which are not requested in the previous rounds of assignment. Then the requested CSs check again whether the total number of new coming proposals and EVs in-suspense exceed the number of available interfaces. If not exceed, cache both new coming EVs and EVs in-suspense in last iteration, otherwise choose $K_{i}$ EVs among the new coming EVs and EVs in-suspense which request $K_{i}$ most amounts of electricity. These chosen EVs become the new EVs in-suspense. The iterations go on until all the charging EVs are in the in-suspense lists of all CSs or EVs which are not suspended by any CSs has been rejected by all CSs in the preference lists. The detailed matching process is illustrated in Algorithm 1.

\section{Cooperative EV Charging AND Discharging in PARKING PATTERN}

Charging scheduling for EVs in driving pattern aims to improve the QoE of charging at charging stations. However, without the service of enough CSs, the ratio of charging requests responded will be decreased, especially in the peak charging hours. Meanwhile, charging served by charging stations may suffer from two issues. First, EVs have to spend extra time to drive to CSs and wait until charging is completed. Second, large scale of EVs charging at CSs simultaneously will lead to overload and greatly impacts the stability of the power grid. Hence, we propose the cooperative charging and discharging scheme in parking pattern to solve these issues. The proposed system is realized with the help of cost-effective 


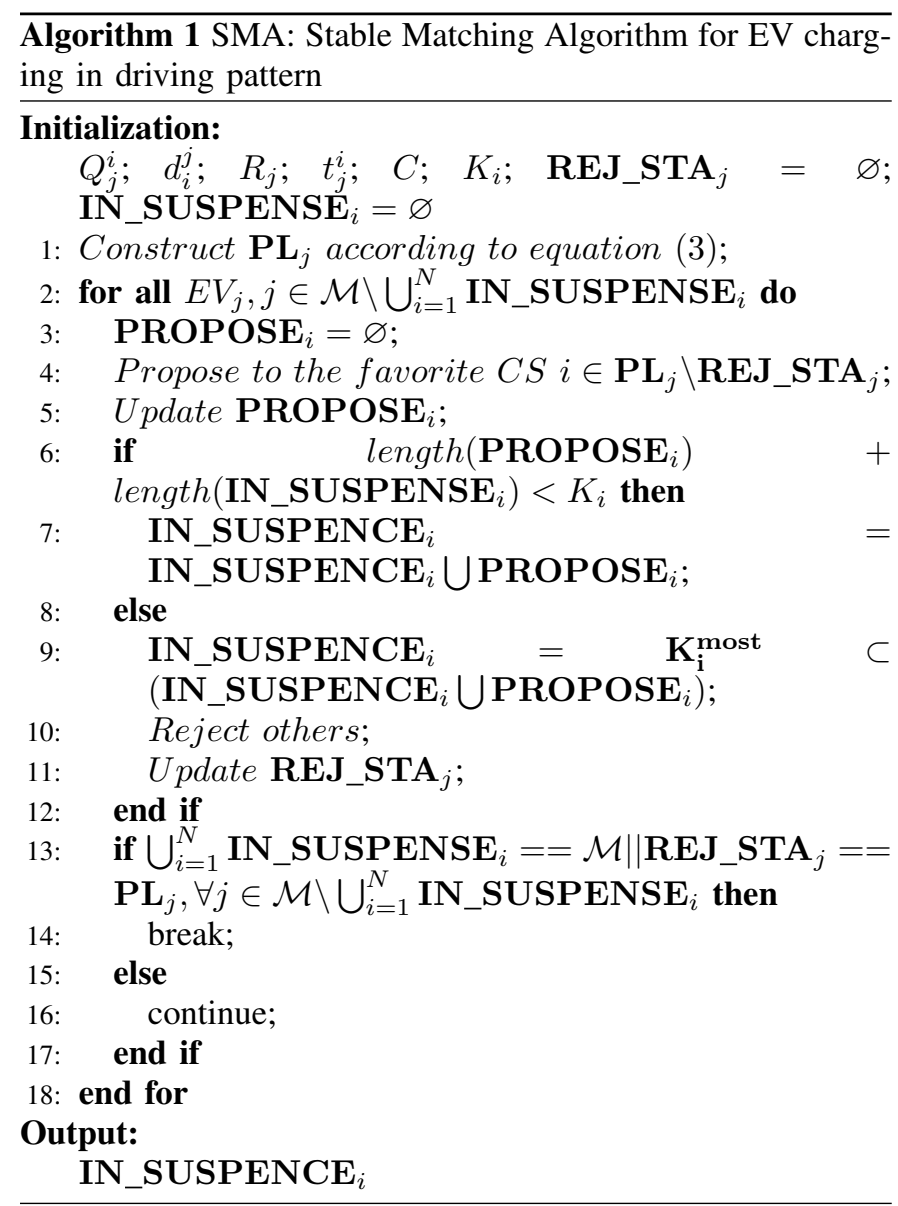

Wireless LAN (WLAN) with multiple access points deployed in the parking lot.

Minimum Reserve Level (MRL) of EV batteries is set by EV drivers to express their range anxiety on the SOC. EVs with battery levels below MRL must be charged before a new trip. Electricity can be drawn from other EVs parking in the same parking lot. EVs which do not have traveling plans until the next day or a couple of days later, posses sufficient electricity and would like to sell can sell electricity to charging $\mathrm{EVs}$ in the same parking lot. In the future, bidirectional charging lines with DC-to-AC converter and AC-to-DC converter can be used to delivery electricity without infrastructure in the underground of every parking lot. The efficiency of DC-to-AC converter can reach around 90\% [21]. And with developing technology, bi-directional charging and discharging can also be implemented through the technology of wireless charging and discharging. As reported, efficiency of wireless charging can be $75 \%$ [16]. Bi-directional chargers and energy management units will be installed on V2G-capable EVs. The energy management units collect and send status information of EVs to an Electricity Exchange Scheduling Sever (EESS) in the parking lot via wireless communication network such as WLAN. After receiving status information of charging EVs and discharging EVs, the EESS determines the pairs of EVs and the amount of electricity for exchange. Since the dwell time of the EVs and the amount of electricity needed and seld are not matched in general, we assume that electricity needed by one charging EV could come from more than one discharging EVs, and one discharging EV can sell electricity to more than one charging EVs.

The main objective for the cooperative electricity exchange is to maximize the trading volume of electricity, while satisfy the constraints of the EVs on the requested or available electricity. A Pareto Optimal Matching Algorithm (POMA) is developed to solve the matching problem between charging EVs and discharging EVs in parking pattern. The problem is formulated as a many-to-many matching model with ties in matching theory.

\section{A. System Model}

Without loss of generality, let $\mathcal{C}$ and $\mathcal{D}$ denote the sets of charging EVs and discharging EVs in the parking lot respectively, where $\mathcal{C}$ and $\mathcal{D}$ are two finite disjoint sets. Each EV reports its SOC, MRL, Minimum Discharging Level (MDL) and discharging willness indicator to the EESS when it enters the parking lot. EVs with SOC lower than the MRL are classified as charging EVs, while EVs with SOC above the MDL are qualified as discharging EVs. The discharging willness indicator is a binary variable, indicating whether a discharging EV is willing to sell its surplus electricity.

Charging EVs are labeled by $c_{i}, i \in\{1,2, \ldots, c\}$, and discharging EVs are labeled by $d_{j}, j \in\{1,2, \ldots, d\}$. Charging EV $c_{i}$ sends its charging request denoted by a three-tuple $\left(p_{c_{i}}, Q_{c_{i}}, t_{c_{i}}\right)$ to the EESS, where $p_{c_{i}}$ represents the unit price of electricity that charging EV $c_{i}$ wants to pay, $Q_{c_{i}}$ indicates the amount of electricity charging EV $c_{i}$ demands, and $t_{c_{i}}$ represents the longest charging duration in which the charging process should be completed. To avoid price alliance in the set of charging EVs, charging requests are first submitted to and stored by the EESS. Then, the EESS forwards charging requests received to all the discharging EVs. After receiving charging requests, discharging $\mathrm{EV} d_{j}$ finds a subset of charging EVs with acceptable charging prices not lower than its reserved price $p_{d_{j}}$. The discharging EV ranks those acceptable charging EVs in a non-increasing order based on $p_{c_{i}}$, and then creats a preference list $\mathbf{P}\left(\mathbf{d}_{\mathbf{j}}\right)$. The preference list is not necessarily strict as the price given by two charging EVs may be equal. Discharging EVs seek profit by participating in cooperative charging and discharging and are indifferent to the electricity buyers. Let $P_{d_{j}}^{u}, u=\left\{1,2, \ldots, U_{d_{j}}\right\}$ denote the indifferent class or tie of charging EVs which offer the $u$ th highest unit price of electricity. The amount of electricity that each discharging EV supplies $Q_{d_{j}}$, the discharging rate $r_{d_{j}}$ and the preference list of discharging EV is submitted to the EESS. Based on the received charging requests and information from discharging EVs, the EESS performs the matching algorithm.

Let $\mathcal{X}$ denote a $C \times D$ allocation matrix with element $x_{i j}=\left\{0, Q_{i j}\right\} . x_{i j}=Q_{i j}$ means discharging EV $d_{j}$ sell $Q_{i j}$ $\mathrm{kWh}$ electricity to charging EV $c_{i}$, while $x_{i j}=0$ means no electricity transaction happens between discharging $\mathrm{EV} d_{j}$ and charging EV $c_{i}$. The utility of discharging EV $d_{j}$ is defined as

$$
U_{d_{j}}=\sum_{c_{i}=c_{1}}^{c_{c}} Q_{i j} \frac{\operatorname{sgn}\left(p_{c_{i}}-p_{d_{j}}\right)}{p_{d_{j}}} \times 100 \%
$$

where $\operatorname{sgn}\left(p_{c_{i}}-p_{d_{j}}\right)$ is the step function. If $p_{c_{i}} \geq p_{d_{j}}$, the value of $\operatorname{sgn}\left(p_{c_{i}}-p_{d_{j}}\right)$ equals to $p_{c_{i}}-p_{d_{j}}$. When $p_{c_{i}}<p_{d_{j}}$, the value of $\operatorname{sgn}\left(p_{c_{i}}-p_{d_{j}}\right)$ equals to 0 . 
The utility of charging EV $c_{i}$ is defined as

$$
U_{c_{i}}=\sum_{d_{j}=d_{1}}^{d_{d}} Q_{i j}
$$

It is noted that the trading volume between charging EV $c_{i}$ and discharging EV $d_{j}$ cannot exceed $Q_{c_{i}}$ and $Q_{d_{j}}$. In addition, electricity exchange process should be completed within the longest charging duration requested by the charging EV. Thus, we have the following constraints, i.e.,

$$
\begin{gathered}
Q_{i j} \leq \min \left(Q_{c_{i}}, Q_{d_{j}}\right) \\
\sum_{c_{i}=c_{1}}^{c_{c}} Q_{i j} \leq Q_{d_{j}}, \sum_{d_{j}=d_{1}}^{d_{d}} Q_{i j} \leq Q_{c_{i}} \\
\frac{Q_{i j}}{r_{d_{j}}} \leq t_{c_{i}}
\end{gathered}
$$

Discharging EVs are incentive rational, if $Q_{i j}$ is positive, this implies that $p_{c_{i}}$ is no less than $p_{d_{j}}$. That is another constraint, i.e.,

$$
p_{c_{i}} \geq p_{d_{j}}, \text { if } Q_{i j}>0
$$

The objective of the proposed model is to maximize the trading volume of electricity, i.e.,

$$
\max _{Q_{i j}} \sum_{d_{j}=d_{1}}^{d_{d}} \sum_{c_{i}=c_{1}}^{c_{c}} Q_{i j}
$$

Thus, the optimization problem is formulated as

$$
\max _{Q_{i j}} \sum_{d_{j}=d_{1}}^{d_{d}} \sum_{c_{i}=c_{1}}^{c_{c}} Q_{i j}
$$

s.t.

$$
\begin{gathered}
p_{c_{i}} \geq p_{d_{j}}, \text { if } Q_{i j}>0 \\
Q_{i j} \leq \min \left(Q_{c_{i}}, Q_{d_{j}}\right) \\
\sum_{c_{i}=c_{1}}^{c_{c}} Q_{i j} \leq Q_{d_{j}}, \sum_{d_{j}=d_{1}}^{d_{d}} Q_{i j} \leq Q_{c_{i}} \\
\frac{Q_{i j}}{r_{d_{j}}} \leq t_{c_{i}}
\end{gathered}
$$

The computational complexity of the optimization problem expressed by Equation (19)-(23) will increase exponentially with the numbers of discharging EVs and charging EVs, and indifference classes exist in the preference lists of discharging EVs. Therefore, we model the optimization problem as a many-to-many matching problem with ties and develop algorithm to solve the problem.

\section{B. Many-to-Many Matching Model with Ties}

In this subsection, we model the cooperative EV charging and discharging problem in parking pattern as a many-tomany matching game with ties. First, we introduce some basic terminologies and then describe the proposed model and algorithm.

Definition 4: Tie [18]

If the order of charging EV $c_{i}$ and charging EV $c_{i^{\prime}}$ in the preference list of discharging EV $d_{j}$ is the same, EV $c_{i}$ and $\mathrm{EV} c_{i^{\prime}}$ belong to the same indifference class or tie to EV $d_{j}$, i.e., $c_{i d_{j}} \simeq c_{i^{\prime}}$.

Definition 5: Pareto Optimal Matching [18]

A matching $\Phi$ is Pareto optimal if there is no other matching in which no EV is worse off and at least one EV is better off.

To find a solution with low computational complexity for the many-to-many matching problem with ties, we map the proposed matching problem into a maximum network flow problem. We construct a network with topology represented by digraph $G=(V, E)$ as shown in Figure 3. The set of network vertexes is denoted by $V=\{$ source, destination $\} \bigcup\left\{d_{j}, j \in\right.$ $\mathcal{D}\} \bigcup\left\{P_{d_{i}}^{u}, j \in \mathcal{D}\right\} \bigcup\left\{c_{i}, i \in \mathcal{C}\right\}$. In addition to the network vertexes representing the actual charging and discharging EVs, two extra vertexes "source" and "destination" are introduced to help solve the matching problem.

The set of edges is denoted by $E=E_{1} \bigcup E_{2} \bigcup E_{3} \bigcup E_{4}$, where

$$
\begin{aligned}
& E_{1}=\left\{\left(\text { source }, d_{j}\right): j \in \mathcal{D}\right\} \\
& E_{2}=\left\{\left(d_{j}, P_{d_{j}}^{u}\right): P_{d_{j}}^{u} \in \mathbf{P}\left(\mathbf{d}_{\mathbf{j}}\right), j \in \mathcal{D}\right\} \\
& E_{3}=\left\{\left(P_{d_{j}}^{u}, c_{i}\right): P_{d_{j}}^{u} \in \mathbf{P}\left(\mathbf{d}_{\mathbf{j}}\right), j \in \mathcal{D}, i \in \mathcal{C}\right\} \\
& E_{4}=\left\{\left(c_{i}, \text { destination }\right): i \in \mathcal{C}\right\}
\end{aligned}
$$

The capacity of each edge in $E_{1} \bigcup E_{2}$ is initialized to be $Q_{d_{j}}$. The capacity of each edge in $E_{4}$ is initialized to be $Q_{c_{i}}$. In the algorithm, $w(e)$ denotes the capacity of $e \in E$, and $N^{\left(d_{j}, P_{d_{j}}^{u}\right)}$ denotes the sub-digraph extracted from $G$ associated with $d_{j}$ and $P_{d_{j}}^{u}$.

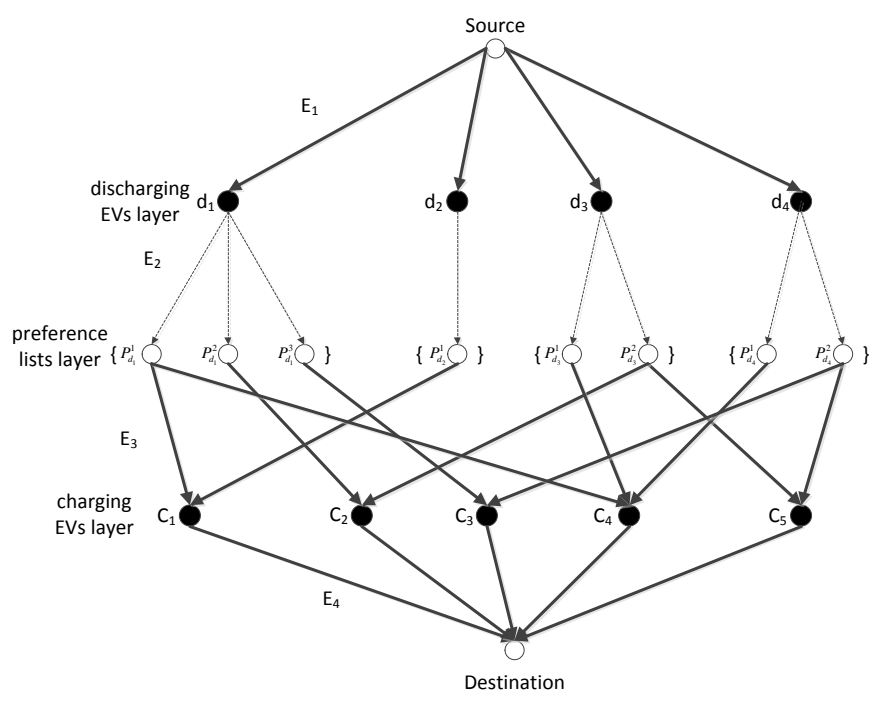

Figure 3: An example for constructing digraph mapped from many-to-many matching problem with ties 


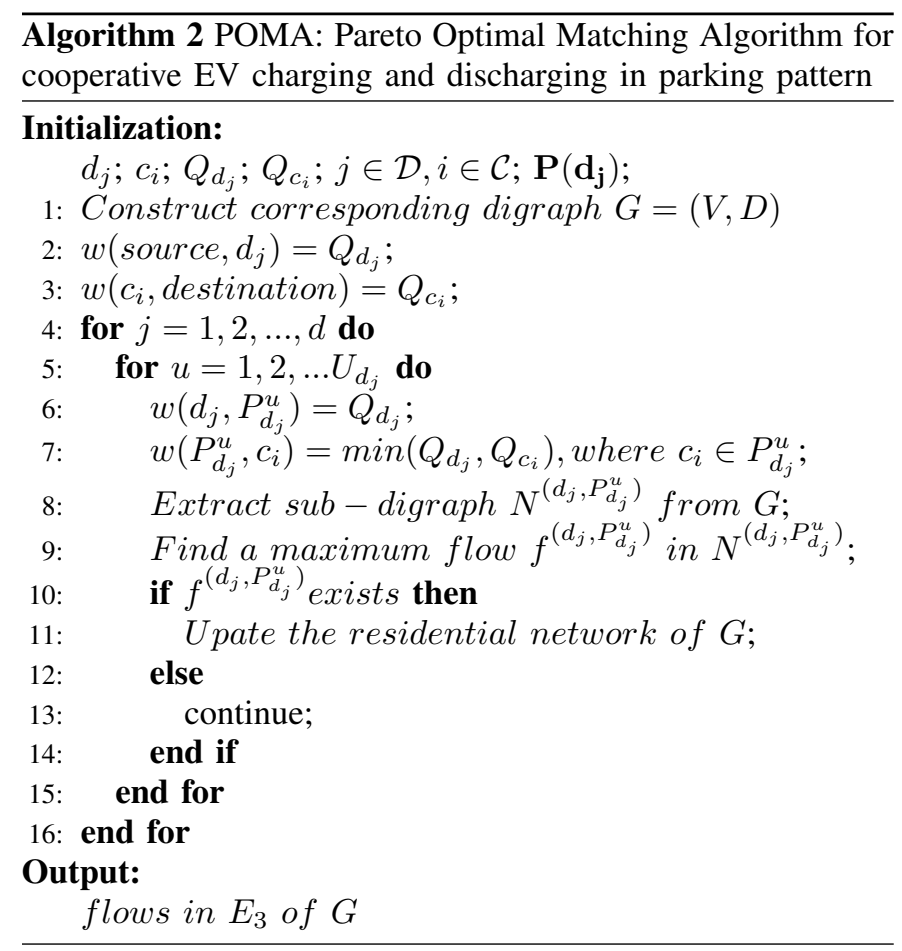

The main process of our proposed algorithm is illustrated in Algorithm 2 and explained as follows. At each stage, a discharging $\mathrm{EV}$ and one of its indifference classes which has not been examined are selected. The sub-digraph with these vertexes is extracted from the original digraph $G$, and a maximum flow in the derived sub-digraph is found. The subdigraphs of discharging EVs which submit their preference lists earlier have higher priority to be selected. Meanwhile, the indifference classes of the selected discharging EV are examined consecutively, according to Theorem 8 in [18] that could guarantee the truthfulness of the matching. The capacity of the edge between the discharging $\mathrm{EV} d_{j}$ and the selected indifferent class is set to be the quota $Q_{d_{j}}$. The capacity of each edge in $E_{3}$ between the selected indifference class and each charging EV included in the indifference class is set to be $\min \left(Q_{d_{j}}, Q_{c_{i}}\right)$ where $c_{i} \in P_{d_{j}}^{u}$. Then the algorithm seeks to find the maximum flow in the extracted sub-digraph $N^{\left(d_{j}, P_{d_{j}}^{u}\right)}$. If a maximum flow does exist, update the residential network of $G$. If not exist, examine the next indifference class. If all the indifference classes of the selected have been examined, then select the next discharging EV. The algorithm terminates until all the indifference classes of the discharging EVs are examined, and the flow in $G$ corresponds to the Pareto-optimal matching between discharging EVs and charging EVs.

The proposed POMA to solve the many-to-many matching problem between charging EVs and discharging EVs in parking pattern is based on the network maximum flow algorithm. The network topology in the maximum flow problem is constructed by two finite sets of charging EVs and discharging EVs as vertexes and preference lists of discharging EVs as edges. Therefore, the POMA is converged. In addition, since the complexity of classic network maximum flow algorithm is $\mathscr{O}(|V||E|$ ) (where $|V|$ represents the number of vertexes in the network topology and $|E|$ represents the number of edges), we derive the complexity of the proposed POMA is $\mathscr{O}\left(c^{2} d\right)$. Although the complexity of the proposed POMA will increase with the number of charging EVs and discharging EVs rapidly, we can divide a large parking lot into many zones when solving the matching problem between charging EVs and discharging EVs in the same parking lot.

\section{Performance Evaluation}

In this section, simulations results are presented for performance evaluation of the proposed SMA and POMA.

For traveling plan-aware charging scheduling in driving pattern, we investigate a $50 \mathrm{~km} \times 50 \mathrm{~km}$ district with $10 \mathrm{CSs}$ randomly distributed in the investigated area. Four types of charging EVs are considered, and their electricity consumption rates can be found in [25]. Each CS is assumed to have 10 charging interfaces. The distance between each EV and each $\mathrm{CS}$ is uniformly distributed in $(0,30] \mathrm{km}$, and the amount of electricity demanded by EV is uniformly distributed in $[10,20] k W h$. The probability that EV may arrive at the CS later than the expected time is set to be 0.2 , and delay cost constant $C$ is set to be 100. Simulation parameters in driving pattern are summarized in Table I. We compare the performance of our proposed SMA with two basic scheduling schemes, the Shortest Distance Priority (SDP) scheme as well as the Only utility of EV Concerned (OEVC) scheme. The SDP scheme assigns the nearest CSs to EVs, while in the OEVC scheme CSs are assigned to EVs purely based on the utilities of EVs and the utilities of CSs in the proposed SMA scheme are ignored. The OEVC scheme is a slightly simplified version of the SMA.

TABLE I: Simulation parameters in driving pattern

\begin{tabular}{|c|c|c|}
\hline Notation & Implication & Value(Distribution) \\
\hline$N$ & Number of CSs & 10 \\
\hline$K_{i}$ & Number of Interfaces of CS $i$ & 10 \\
\hline$Q_{j}^{i}$ & Amount of Electricity Needed & $U[10,20](\mathrm{kWh})$ \\
\hline$d_{j}^{i}$ & Distance from EV $j$ to CS $i$ & $U(0,30](\mathrm{km})$ \\
\hline$R_{j}$ & Electricity Consumption Rate & $\begin{array}{c}\text { randomly choose from } \\
(0.121,0.15,0.16,0.21) \\
(\mathrm{kWh} / \mathrm{km})\end{array}$ \\
\hline$p\left(t_{j}^{i}>t_{j}\right)$ & Probability of Delay & 0.2 \\
\hline$C$ & Delay Cost & 100 \\
\hline$\beta$ & Weight Parameter & 1 \\
\hline
\end{tabular}

Figure 4 shows the gap between the global optimum and the proposed SMA. Due to the high computational complexity to get the global optimal solution, we run a simulation with small scale of CSs and charging EVs. In the simulation, 3 CSs offer charging service to EVs and each CS has 3 charging interfaces. The average system utility that the proposed SMA can reach is close to the global optimum. However, the proposed SMA is a decentralized solution, while to get the global optimal solution by exhaustive search the computational complexity is $\mathscr{O}\left(N \sum_{i=1}^{N} K_{i}\right)$.

Figure 5 shows the system utilities in driving pattern with the proposed SMA comparing to the SDP scheme and the OEVC scheme. It can be observed that the proposed SMA has the highest average system utility among the three schemes. The performance gain of the average system utility by the SMA is up to $47.4 \%$ comparing to the SDP scheme, and $3.37 \%$ over the OEVC scheme when the number of charging 


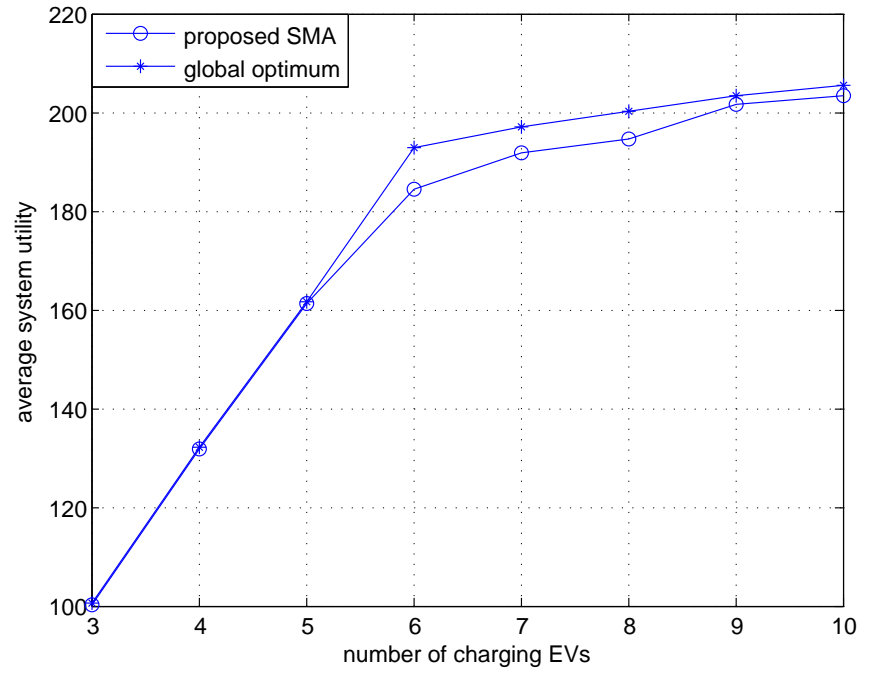

Figure 4: Average system utility under the proposed SMA compared to the global optimum

requests is more than the total number of interfaces of all CSs. When there are sufficient interfaces at CSs, the average system utility of SMA and OEVC are almost the same, and this is because all most every charging requests are satisfied. However, when substantial charging requests need to be responded, the proposed SMA gives priority to charging request demanding for more electricity which improves the utilities of CSs and increases the average system utilities.

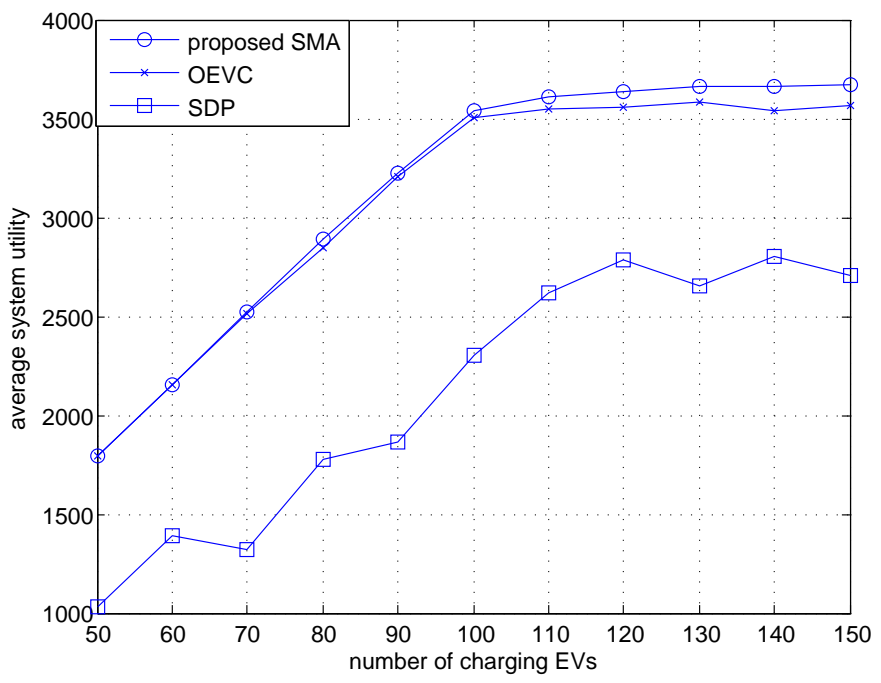

Figure 5: Average system utility under different schemes

For cooperative EV charging and discharging in parking pattern, we consider electricity discharged from discharging EVs is used to charge EVs directly through the bidirectional charging lines laid in the underground of parking lots. The amount of electricity demanded by charging EVs is uniformly distributed in $[10,20] \mathrm{kWh}$, and the amount of electricity offered by discharging EVs is uniformly distributed in $[5,15] \mathrm{kWh}$. For tractability, the bidding unit prices of electricity of charging EVs and the reserved unit prices of electricity of discharging EVs are randomly chosen from a finite
TABLE II: Simulation parameters in parking pattern

\begin{tabular}{|c|c|c|}
\hline Notation & Implication & Value(Distribution) \\
\hline$Q_{c_{i}}$ & $\begin{array}{c}\text { Amount of } \\
\text { Electricity Needed }\end{array}$ & $U[10,20](k W h)$ \\
\hline$Q_{d_{j}}$ & $\begin{array}{l}\text { Amount of } \\
\text { Electricity Discharged }\end{array}$ & $U[5,15](k W h)$ \\
\hline$p_{c_{i}}$ & $\begin{array}{l}\text { Unit Price of Electricity } \\
\text { Charging EV } c_{i} \\
\text { Wants to Pay }\end{array}$ & $\begin{array}{c}\text { randomly } \\
\text { choose from } \\
(0.3,0.4,0.5,0.6,0.7,0.8)\end{array}$ \\
\hline$p_{d_{j}}$ & $\begin{array}{c}\text { Reserved Unit Price of } \\
\text { Electricity } \\
\text { Disharging EV } d_{j} \\
\text { Would Sell }\end{array}$ & $\begin{array}{c}\text { randomly } \\
\text { choose from } \\
(0.3,0.4,0.5,0.6,0.7,0.8)\end{array}$ \\
\hline
\end{tabular}

price vector $(0.3,0.4,0.5,0.6,0.7,0.8)$ according to electricity tariff in [25]. Simulation parameters in parking pattern are summarized in Table II. We compare the performance of our proposed POMA with a Random Scheduling (RS) algorithm in which charging EVs are randomly assigned to discharging EVs. We also study how the trading volume of electricity changes as the number of charging EVs and discharging EVs increases. Moreover, we show the transaction prices of electricity with the proposed POMA.

Figure 6 compares the trading volume of electricity with the POMA and RS algorithm over the increasing number of charging EVs and discharging EVs. The trading volume of electricity with the proposed POMA is larger than the RS scheme, and increases with the number of EVs. It proves the effectiveness of our proposed scheme, and shows that the proposed POMA can have a significant impact on the reliability of the power grid. The more electricity exchanged between charging EVs and discharging EVs in peak hours of the power grid, the less electricity to be supplied from the grid.

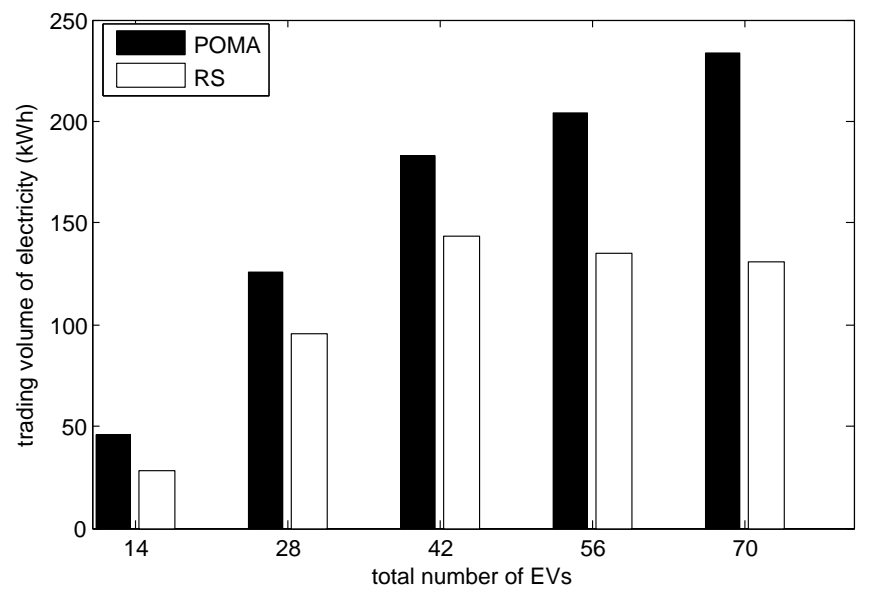

Figure 6: Trading volume of electricity under different schemes

Figure 7 further shows the benefits to the power grid by encouraging cooperative EV charging and discharging. The peak-to-valley value of electric load is greatly reduced. $500,000 \mathrm{EVs}$ are investigated in the simulation scenario, and the peak-to-valley value of electric load of the grid falls from $6.05 \mathrm{GW}$ to $5.26 \mathrm{GW}$.

Figure 8 shows the transaction price of electricity from the assigned discharging EVs. The transaction price is always no 


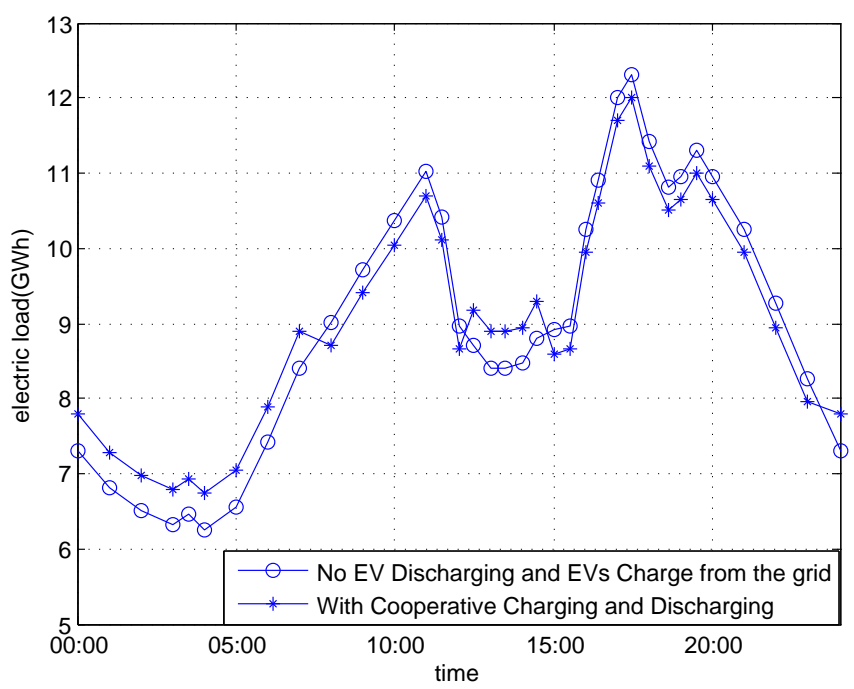

Figure 7: Electric load curve under difference schemes

less than the reserved price with the proposed POMA, which offers incentives to EVs to sell electricity when they have surplus electricity and have no imminent travelings.

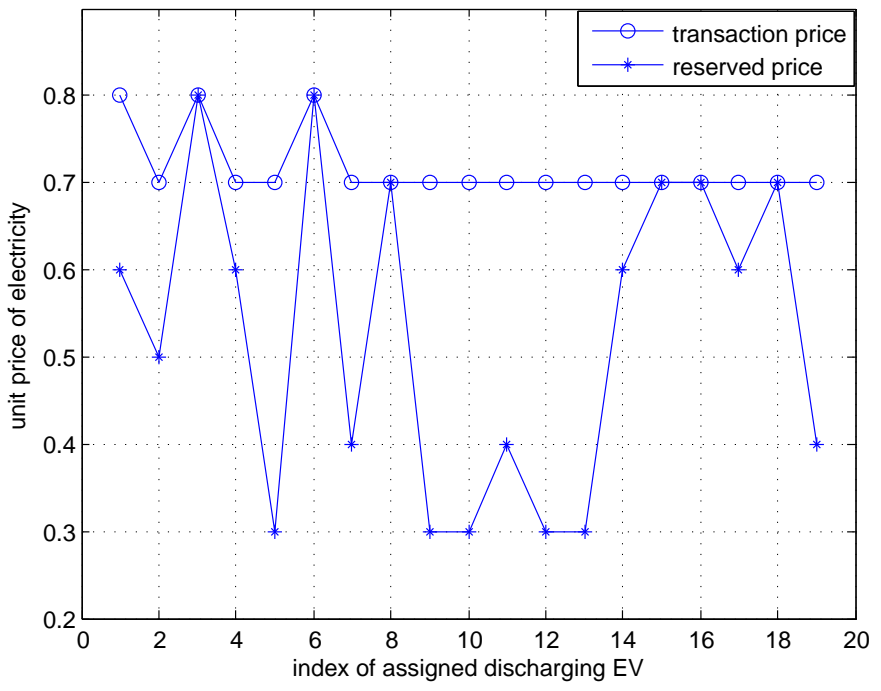

Figure 8: Difference between transaction price and reserved price for assigned discharging EVs

\section{CONCLUSION}

QoE of using EVs has significant impact on the development of V2G system. In this paper, we design the traveling plan-aware scheduling scheme for EV charging in driving pattern and investigate cooperative EV charging and discharging in parking pattern. A matching theoretic framework is applied, where the problem in driving pattern is formulated as a many-to-one matching problem, and the problem in parking pattern is formulated as a many-to-many matching problem with ties. We propose the Stable Matching Algorithm (SMA) to find a stable matching between charging EVs and CSs and the Pareto Optimal Matching Algorithm (POMA) to find a Pareto optimal matching between charging EVs and discharging EVs in parking pattern. Simulation results show the proposed SMA can improve the system utility and increase the QoE of charging EVs in driving pattern. In parking pattern, the proposed POMA is helpful to enhance the reliability of the power grid. Moreover, incentives are offered to encourage EVs to participate in cooperative charging and discharging system actively.

\section{REFERENCES}

[1] M. F. Jung, D. Sirkin and T. M. Gur, "Displayed Uncertainty Improves Driving Experience and Behavior: The Case of Range Anxiety in an Electric Car", Proceedings of the 33rd Annual ACM Conference on Human Factors in Computing Systems, April 2015.

[2] W. Kempton and T. Jasna, "Vehicle to Grid Fundamentals: Calculating Capacity and Net Revenue, Journal of Power Sources, vol. 144, no. 1, pp. 268-279, June 2005 .

[3] J. V. Roy, N. Leemput, F. Geth, R. Salenbien, J. Buscher and J. Driesen "Apartment Building Electricity System Impact of Operational Electric Vehicle Charging Strategies", IEEE Transaction on Sustainable Energy, vol. 5, no. 1, pp. 264-272, January 2014.

[4] Q. Huang, Q. S. Jia, Z. Qiu, X. Guan and G. Deconinck, "Matching EV Charging Load With Uncertain Wind: A Simulation-Based Policy Improvement Approach", IEEE Transaction on Smart Grid, vol. 6, no. 3, pp. 1425-1433, May 2015.

[5] N. Korolko and Z. Sahinoglu, "Robust Optimization of EV Charging Schedules in Unregulated Electricity Markets", IEEE Transaction on Smart Grid, vol. 99, no. 99, pp. 1-9, September 2015.

[6] L. Rao and J. Yao, "SmartCar: Smart Charging and Driving Control for Electric Vehicles in the Smart Grid", IEEE Global Communications Conference (GLOBECOM), December 2014.

[7] J. Donadee and M. D. Ilic, "Stochastic Optimization of Grid to Vehicle Frequency Regulation Capacity Bids", IEEE Transaction on Smart Grid, vol. 5, no. 2, pp. 1061-1069, March 2014.

[8] C. Luo, Y. F. Huang, and V. Gupta, "A Consumer Behavior Based Approach to Multi-Stage EV Charging Station Placement", IEEE 81st Vehicular Technology Conference (VTC Spring), May 2015.

[9] J. Tan and L. Wang, "Real-Time Charging Navigation of Electric Vehicles to Fast Charging Stations: A Hierarchical Game Approach", IEEE Transaction on Smart Grid, vol. 99, no. 99, pp. 1-11, August 2015.

[10] N. Z. Xu and C. Y. Chung, "Challenges in Future Competition of Electric Vehicle Charging Management and Solutions", IEEE Transaction on Smart Grid, vol. 6, no. 3, pp. 1323-1331, April 2015.

[11] F. Rassaei, W. S. Soh and K. C. Chua, "Demand Response for Residential Electric Vehicles With Random Usage Patterns in Smart Grids", IEEE Transaction on Sustainable Energy,, vol. 6, no. 4, pp. 1367-1376, October 2015.

[12] Y. Gu, W. Saad, M. Bennis, M. Debbah and Z. Han, "Matching Theory for Future Wireless Networks: Fundamentals and Applications", IEEE Communications Magazine, vol. 53, no. 5, pp. 52-59, May 2015.

[13] Q. Wang, S. Leng, H. Fu and Y. Zhang, "An IEEE 802.11p-based Multichannel MAC Scheme with Channel Coordination for Vehicle Ad Hoc Networks", IEEE Transactions on Intelligent Transportation Systems, vol. 13, no. 2, pp. 449-458, June 2012.

[14] C. Shao, S. Leng, Y. Zhang, A. Vinel and M. Jonsson "Performance Analysis of Connectivity Probability and Connectivity-Aware MAC Protocol Design for Platoon-Based VANETs", IEEE Transactions on Vehicle Technology, December 2015.

[15] A. E. Roth, "The college admissions problem is not equivalent to the marriage problem", Journal of Economic Theory, vol. 36, no. 2, pp. 277-288, March 1985.

[16] Wireless Bus Charging. [Online]. Available: http://kojects.com/2013/08/ 13/wireless-charging-electric-bus-in-gumi/

[17] D. Gale and L. S. Shapley, " College Admissions and the Stability of Marriage", The American Mathematical Monthly, vol. 69, no. 1, pp. 9-15, January 1962.

[18] K. Cechlarova, P. Eirinakis, T. Fleiner, D. Magos, D. Manlove, I. Mourtos, E. Ocelakova and B. Rastegari, " Pareto Optimal Matchings in Manyto-Many Markets with Ties", Springer Berlin Heidelberg, pp. 27-39, December 2015.

[19] P. Krysta, D. Manlove, B. Rastegari and J. Zhang, "Size Versus Truthfulness in the House Allocation Problem", 15th ACM Conference on Economics \& Computation, June 2014.

[20] C. H. Papadimitriou, and K. Steiglitz, "Combinatorial optimization: algorithms and complexity", Prentice Hall, 1998.

[21] H. B. Ertan, E. Dogru and A. Yilmaz, "Comparison of Efficiency of Two dc-to-ac Converters for Grid Connected Solar Applications", IEEE International Conference on Optimization of Electrical and Electronic Equipment, May 2012. 
[22] H. Qin and W. Zhang, "Charging scheduling with minimal waiting in a network of electric vehicles and charging stations", Eighth ACM International Workshop on Vehicular Inter-networking, January 2011.

[23] V. Razo and H. A. Jacobsen, "Smart Charging Schedules for Highway Travel with Electric Vehicles", IEEE Transactions on Transportation Electrification, vol. 1, no. 99, pp. 1-14, April 2016.

[24] C. Bodet, A. Schulke, K. Erickson and R. Jablonowski, "Optimization of charging infrastructure usage under varying traffic and capacity conditions", IEEE International Conference on Smart Grid Communications, November 2012.

[25] M. Zeng, S. Leng and Y. Zhang, "Power Charging and Discharging Scheduling for V2G Networks in the Smart Grid", IEEE International Conference on Communications (ICC) Workshops, June 2013.

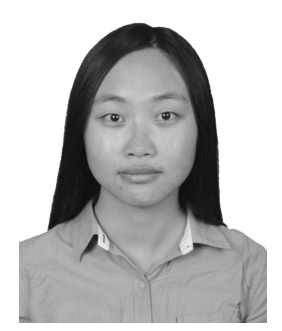

Ming Zeng is currently working toward the Ph.D. degree at the School of Communication and Information Engineering, University of Electronic Science and Technology of China. She focuses on the resource allocation issue in vehicle-to-grid system and wireless communication networks. Her research interests include mechanism design based on auction theory and matching theory.

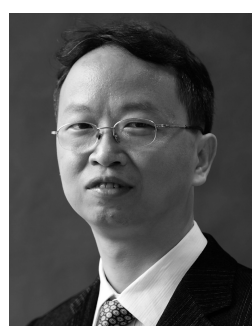

Supeng Leng (M'06) is a Professor in the School of Communication and Information Engineering, University of Electronic Science and Technology of China (UESTC). He received his Ph.D. degree from Nanyang Technological University (NTU), Singapore. He has been working as a Research Fellow in the Network Technology Research Center, NTU. His research focuses on resource, spectrum, energy, routing and networking in broadband wireless access networks, vehicular networks, internet of things, next generation mobile networks, and smart grid. He published over 100 research papers in recent years. He serves as an organizing committee chair and TPC member for many international conferences, as well as a reviewer for over 10 international research journals.

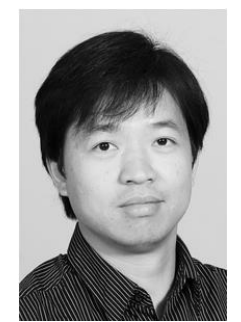

Yan Zhang is full-time Full Professor at University of Oslo, Norway. He is also Chief Scientist at Simula Research Laboratory, Norway. He received a PhD degree in School of Electrical and Electronics Engineering, Nanyang Technological University, Singapore. He is an associate editor or on the editorial board of a number of well-established scientific international journals. He is currently serving the Book Series Editor-in-Chief for the book series on "Wireless Networks and Mobile Communications" (CRC Press). He serves as organizing committee chairs and technical program committee for many international conferences. He has received 8 Best Paper Awards. His current research interests include: wireless networks leading to $5 \mathrm{G}$, and cyber-physical systems (e.g., smart grid, healthcare, transport). He is a senior member of IEEE, IEEE ComSoc, Computer Society, PES and IEEE VTS. He is a Fellow of IET.

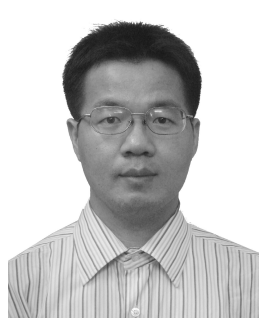

Jianhua He is a Lecturer at the School of Engineering and Applied Science, Aston University, UK. He received a BEng and MEng degree on Electronic Engineering from Huazhong University of Science and Technology (HUST), China, and a Ph.D. degree on Communications Engineering from Nanyang Technological University, Singapore, in 1995, 1998, and 2002, respectively. Dr He joined HUST in 2001 as an Associate Professor. He was with University of Bristol from 2004 to 2006 and with University of Essex in 2007. His main research interests include $5 \mathrm{G}$ and beyond technologies, machine to machine communications, Internet of things system and technologies (e.g. for small cities and intelligent transport) and big data analytics. He has authored or co-authored over 150 technical papers in major international journals and conferences. Dr. He is a Senior Member of IEEE. He is or has been editor/guest editor of a number of journals, TPC chair and TPC member of many international conferences, e.g., IEEE ICC and Globecom. 